\title{
Reporter-Embedded SERS Tags from Gold Nanorod Seeds: Selective Immobilization of Reporter Molecules at the Tip of Nanorods
}

\author{
Yuan Wang, ${ }^{\dagger \neq}$ Yunqing Wang, ${ }^{*}$, Wenhai Wang, $^{*}$ Kaoxiang Sun, ${ }^{* \dagger}$ and Lingxin Chen $*,+$
}

${ }^{\dagger}$ School of Pharmacy, Collaborative Innovation Center of Advanced Drug Delivery System and Biotech Drugs in Universities of Shandong, Key Laboratory of Molecular Pharmacology and Drug Evaluation (Yantai University), Ministry of Education, Yantai University, Yantai 264005, China

${ }^{\ddagger}$ Key Laboratory of Coastal Environmental Processes and Ecological Remediation, Yantai Institute of Coastal Zone Research, Chinese Academy of Sciences, Yantai 264003, China

\section{Supporting Information}

ABSTRACT: Reporter-embedded (RE) tags are a new generation of sensitive, stable surface enhanced Raman scattering (SERS) tags with Raman reporters embedded between gold nanoparticle (NP) cores and gold (or silver) shells. Most of the reported RE tags have been designed using Au nanospheres as a seed material. Herein, we investigated the synthesis and SERS properties of AuNR/reporter/Ag tags by using gold nanorod (AuNR) seeds with anisotropic physical and optical features. Several highlighted points were discovered, including the following: (1) The cetyltrimethylammonium bromide (CTAB) layer induced the coexistence of chemically and physically adsorbed Raman reporters on

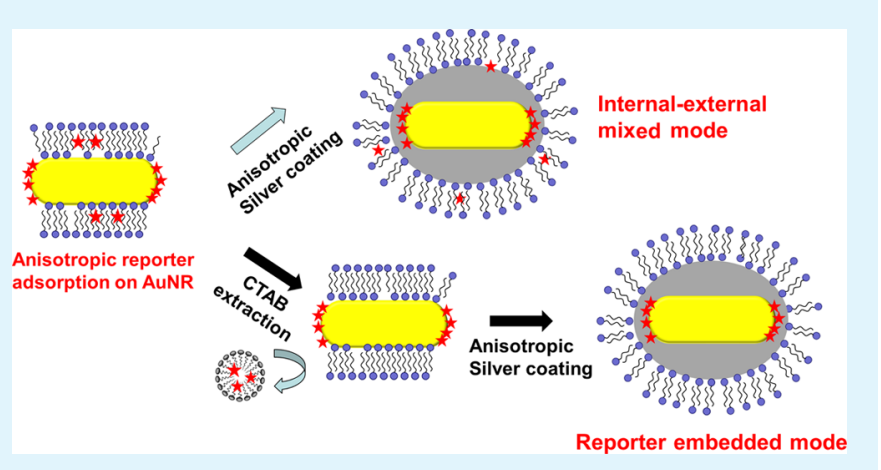
AuNR. Conventional washing of the AuNR-reporter complex with water results in the formation of an "internal-external" mixed tag. To obtain a "pure" RE structure, an additional extraction step involving a CTAB solution was essential. (2) The anisotropic distribution of $\mathrm{CTAB}$ on AuNR resulted in the preference of the Raman reporters to adsorb to the hotspot at the AuNR tip, which made it a perfect match for improving the SERS signal of the tag. (3) An anisotropic silver coating occurred with the shell thickness on the AuNR side growing much faster than the shell thickness at the tip. This feature ensured that the tag grew to a suitable size with enough silver for SERS enhancement without shadowing the effective Raman reporters at the tip too much. (4) RE tags showed better in vitro and in vivo signal stabilities compared with their external labeling counterparts. Moreover, a novel $\mathrm{pH}$-sensitive SERS peak test was proposed by using 4-mercaptobenzoic acid as the Raman reporter to verify thin coverage by a silver layer. We believe this tag can be broadly applied for molecular detection and bioimaging, and the proposed preparation and structure verification methods can provide universal guidance in the design of novel RE tags.

KEYWORDS: gold nanorod, SERS tags, internal standard, core-shell nanoparticle, anisotropic property

\section{INTRODUCTION}

Surface-enhanced Raman scattering (SERS) tags have been considered as attractive optical nanoprobes owing to their potential in chemical sensing and biological imaging applications. $^{1-3}$ Conventional SERS tags are prepared by attaching Raman reporters to the surface of noble metal nanoparticles (NPs), thereby creating a known SERS spectrum of the Raman reporter. In recent years, interest in synthesizing reporter-embedded (RE) SERS tags with Raman reporters embedded between the $\mathrm{Au}$ core and $\mathrm{Au}$ (or $\mathrm{Ag}$ ) shell has grown. ${ }^{4-8} \mathrm{RE}$ tags have superior optical features relative to conventional SERS tags including: (1) They have strongly enhanced Raman signals due to the built-in hotspots. (2) They have a better signal stability because the metal shell covering can prevent the possible signal fluctuations induced by desorption or structural changes of the Raman molecules. (3) They can be used as novel SERS substrates for reliable SERS analysis by using the RE signal as an internal standard. The quantitative analysis of target molecules over a large concentration range can be realized from the linear response of the internal standard intensity versus signal intensity of target molecules covering on the tag surface. ${ }^{9-11}$

Two synthesis strategies of RE tags have been proposed until now. One is to directly deposit Ag or Au shells on seed NPs functionalized with small thiol molecules. ${ }^{5,11}$ The other is to form nanomatryoshka structures in which an insulation matrix layer (such as DNA, ${ }^{7,8,12-15}$ polymer, ${ }^{6}$ and dithiol molecules $^{4,16,17}$ ) with Raman-active parts is applied to modify the seed NPs before outer shell growth. In most RE tags, gold nanospheres (NSs) or nanostars are selected as seed NPs. RE tags prepared from gold nanorod (AuNR) seeds have rarely been investigated.

Received: April 9, 2016

Accepted: October 3, 2016

Published: October 3, 2016 
As a famous nanomaterial, AuNR has drawn broad interest for the fabrication of conventional SERS tags with theranostic functions $^{18,19}$ or the application as SERS substrates for molecular detections. ${ }^{20-22}$ We believed that AuNR-based RE tags also deserved deep investigation because of special anisotropic features of AuNR. On the one hand, the SERS enhancement ability at the tips of the AuNRs was much stronger than that of the sides. ${ }^{23,24}$ Simultaneously, the AuNR exhibited a tip-selective Raman reporter adsorption behavior. $^{25,26}$ The matched properties would be advantageous to improve the sensitivity of the RE tags. On the other hand, the silver shell of AuNR/Ag core/shell NPs evolved from homogeneous to special anisotropic coatings on AuNRs with increasing amounts of silver. ${ }^{27}$ This would make the SERS signal evolution of the tags complicated. Moreover, AuNR was capped by a dense long-chain cetyltrimethylammonium bromide (CTAB) bilayer, which led to difficulties in Raman reporter adsorption. The effect of capping agents of seed NPs on the formation of RE tags was still unclear.

To address these questions, we investigated the synthesis procedure and SERS properties of AuNR/reporter/Ag coreshell RE tags. Thiol-containing small molecules were selected as Raman reporters and attached on the surface of the AuNRs. Subsequently, a silver shell was deposited onto the seed to complete the AuNR-core/Ag-shell nanostructure. It was found that chemically and physically adsorbed reporters always coexist on AuNR due to the capacity of CTAB bilayers to hold molecules. The clearance of the physically adsorbed reporters by CTAB solution was a crucial step to obtain a "pure" RE structure. RE tags showed high sensitivity because of both tipselective Raman reporter adsorption feature and anisotropic silver shell coating property of AuNRs. Taking advantage of the silver shell coverage, $\mathrm{RE}$ tags showed better in vitro and in vivo signal stability compared with conventional "external" tags with Raman reporters adsorbed on the outer surface of NPs. Moreover, a novel method was proposed to verify the Raman reporter encapsulation by monitoring the Raman profile changing of a $\mathrm{pH}$-sensitive Raman reporter (4-mercaptobenzoic acid, MBA) in different $\mathrm{pH}$ conditions.

\section{EXPERIMENTAL SECTION}

Materials. Chloroauric acid $\left(\mathrm{HAuCl}_{4}\right)$, silver nitrate $\left(\mathrm{AgNO}_{3}\right)$, sodium borohydride $\left(\mathrm{NaBH}_{4}\right)$, sodium hydroxide $(\mathrm{NaOH})$, L-ascorbic acid (AA), hydroxylamine hydrochloride $\left(\mathrm{NH}_{2} \mathrm{OH} \cdot \mathrm{HCl}\right)$, polyvinylpyrrolidone (PVP, MW 58 000), and absolute ethyl alcohol were purchased from Sinopharm Chemical Reagent Co., Ltd. Cetyltrimethylammonium bromide $(\mathrm{CTAB})$, cetyltrimethylammonium chloride (CTAC), glutathione, 1-naphthalenethiol (NAT), 4-nitrothiophenol (4-NT), 4-aminothiophenol (4-ATP), and 4-mercaptobenzoic acid (MBA) were obtained from Sigma-Aldrich. mPEG-SH (MW 2000) was obtained from Yare Biotech. Deionized water was used in all of the experiments.

Characterization. TEM images were acquired on a JEM-1400 transmission electron microscope (JEOL, Japan). SEM images of the samples were obtained by using a field-emission microscope (Hitachi S-4800, Japan), and UV/vis/NIR absorption spectra were recorded on a Thermo Scientific NanoDrop 2000/2000C spectrophotometer. The hydrodynamic diameter was measured on a Zetasizer NanoZS90 (Malvern Instruments, Malvern, U.K.). The SERS spectra were recorded by using a DXR Raman Microscope (Thermo Scientific, USA). A $632.8 \mathrm{~nm}, 1 \mathrm{~mW} \mathrm{He}-\mathrm{Ne}$ laser was focused by a microscope with $10 \times$ and $50 \times$ objective lenses for sample solution and cell measurement, respectively.

Preparation of AuNRs. AuNRs were synthesized by using the seed-mediated growth method. ${ }^{28}$ Briefly, the seed solution was prepared by reducing $\mathrm{HAuCl}_{4}(0.5 \mathrm{mM}, 2 \mathrm{~mL})$ in CTAB $(0.2 \mathrm{M}, 2$ $\mathrm{mL}$ ) with freshly prepared ice-cold $\mathrm{NaBH}_{4}(10 \mathrm{mM}, 0.24 \mathrm{~mL})$. After 2 $\mathrm{h}, 3.6 \mathrm{~mL}$ of the resultant seed solution was added into a growth solution of $\mathrm{HAuCl}_{4}(23 \mathrm{mM}, 13 \mathrm{~mL})$, CTAB $(0.2 \mathrm{M}, 200 \mathrm{~mL})$, $\mathrm{AgNO}_{3}(4 \mathrm{mM}, 11.2 \mathrm{~mL})$, and $\mathrm{AA}(80 \mathrm{mM}, 5 \mathrm{~mL})$. The mixture was stored overnight at $27-30{ }^{\circ} \mathrm{C}$.

Raman Reporter Incubation. The as-prepared AuNRs (1 mL) were centrifuged twice at $9200 \mathrm{rpm}$ for $15 \mathrm{~min}$ and redispersed in 950 $\mu \mathrm{L}$ of water. Then, $50 \mu \mathrm{L}$ of Raman reporter ethanol solution was added to achieve a final concentration of $5 \times 10^{-5} \mathrm{M}$ for NT, NAT, 4ATP, and $10^{-5} \mathrm{M}$ for MBA. The mixture was allowed to react for $2 \mathrm{~h}$ at room temperature.

Preparation of "Internal-External" Mixed Tags. The AuNRreporter mixture was centrifuged at $9100 \mathrm{rpm}$ for $15 \mathrm{~min}$, washed twice with water, and then redispersed in $1 \mathrm{~mL}$ of water. The absorbance at $750 \mathrm{~nm}$ was approximately 1.7 . One milliliter of CTAB $(0.2 \mathrm{M})$ was added to $300 \mu \mathrm{L}$ of this seed solution, followed by the addition of water until the total volume was $6 \mathrm{~mL}$. Then, $20 \mu \mathrm{L}$ of AA $(0.1 \mathrm{M})$ and 50 or $100 \mu \mathrm{L}$ of $\mathrm{AgNO}_{3}(10 \mathrm{mM})$ were added in sequence at room temperature while stirring. Finally, the $\mathrm{pH}$ of the mixture was adjusted to approximately 10 by adding $\mathrm{NaOH}(0.1 \mathrm{M})$. The solution color immediately started to change from pink to blue or red, which indicated the formation of a $\mathrm{Ag}$ layer on AuNR. The mixture was stirred for $20 \mathrm{~min}$ to complete the reaction. For obtaining the tags prepared from 300,700 , or $1100 \mu \mathrm{L}$ of $\mathrm{AgNO}_{3}$, an additional $20 \mu \mathrm{L}$ of AA, $200 \mu \mathrm{L}$ of $\mathrm{AgNO}_{3}$, and $100 \mu \mathrm{L}$ of $\mathrm{NaOH}$ was added to the above reaction mixture containing $100 \mu \mathrm{L}$ of $\mathrm{AgNO}_{3}$ for one, three, and five rounds, respectively.

Preparation of RE Tags. The AuNR-reporter mixture was centrifuged at $9100 \mathrm{rpm}$ for $15 \mathrm{~min}$ and washed four times with 0.2 $\mathrm{M}$ CTAB solution. The seed NPs were then redispersed in $1 \mathrm{~mL}$ of water. The absorbance at $750 \mathrm{~nm}$ was around 1.0. One milliliter of CTAB $(0.2 \mathrm{M})$ was added to $520 \mu \mathrm{L}$ of this seed solution, followed by the addition of water until the total volume was $6 \mathrm{~mL}$. The silver shell growth was performed using the same procedure as that for the "internal-external" mixed tags.

Preparation of Conventional "External" Tags. The as-prepared AuNRs $(1 \mathrm{~mL})$ were centrifuged twice for $15 \mathrm{~min}$ at a speed of 9200 rpm and redispersed in $1 \mathrm{~mL}$ of water. Then, AuNR/Ag NPs were synthesized according to the aforementioned silver coating procedure. The resulting AuNR/Ag NPs were centrifuged at $7000 \mathrm{rpm}$ for $15 \mathrm{~min}$ and redispersed in $6 \mathrm{~mL}$ of water, followed by the addition of $50 \mu \mathrm{L}$ of NT $(1 \mathrm{mM})$. The mixture was allowed to react for $2 \mathrm{~h}$.

Preparation of "mPEG on AuNR Tip" RE Tags. AuNRs ( $1 \mathrm{~mL})$ were centrifuged for $15 \mathrm{~min}$ at a speed of $9200 \mathrm{rpm}$. Then, $50 \mu \mathrm{L}$ of mPEG-SH $(20 \mathrm{mM})$ was added and the mixture was vortexed for $30 \mathrm{~s}$. The mixture was allowed to react for $2 \mathrm{~h}$. Finally, PEG-modified AuNR was centrifuged at $9200 \mathrm{rpm}$ for $15 \mathrm{~min}$ to remove excess mPEG-SH and redispersed in water $(950 \mu \mathrm{L})$. Then, Raman reporter incubation, washing with $\mathrm{CTAB}$ solution, and the silver shell coating process were performed in a similar manner as that described for the preparation of RE tags.

Preparation of Au Nanosphere (NS) Seeded RE Tags. Conventional AuNSs were used as seeds to prepare RE tags with the same manner for SERS property comparison. AuNSs were prepared followed by a previously reported work. ${ }^{29}$ Briefly, freshly prepared $\mathrm{NaBH}_{4}$ solution $(1 \mathrm{mM}, 0.6 \mathrm{~mL})$ was rapidly added into a thoroughly mixed $10 \mathrm{~mL}$ of aqueous solution containing $\mathrm{HAuCl}_{4}(0.25$ $\mathrm{mM})$ and CTAB $(0.1 \mathrm{M})$. A brown solution immediately formed upon the introduction of $\mathrm{NaBH}_{4}$, which indicated the formation of $\mathrm{Au}$ clusters. The formed Au clusters was stirred at a speed of $300 \mathrm{rpm}$ for $2 \mathrm{~min}$, and then kept undisturbed at $27^{\circ} \mathrm{C}$ for $3 \mathrm{~h}$. Aqueous solutions of CTAC (0.2 M, $2 \mathrm{~mL}), \mathrm{AA}(0.1 \mathrm{M}, 1.5 \mathrm{~mL})$, and Au clusters $(20$ or $50 \mu \mathrm{L})$ were mixed, followed by the injection of $\mathrm{HAuCl} 4$ solution $(0.5$ $\mathrm{mM}, 2 \mathrm{~mL}$ ). The reaction was allowed to continue at $27^{\circ} \mathrm{C}$ for 15 min. The product was AuNS with a diameter of 16 and $10 \mathrm{~nm}$, respectively. The product (AuNS, $10 \mathrm{~nm}$ ) was collected by centrifugation at $14000 \mathrm{rpm}$ for $30 \mathrm{~min}$, and then washed with water once. Then, CTAC $(0.1 \mathrm{M}, 2 \mathrm{~mL}), \mathrm{AA}(10 \mathrm{mM}, 130 \mu \mathrm{L})$, and the $10 \mathrm{~nm}$ seeds $(10 \mu \mathrm{L})$ were mixed, followed by dropwise addition 


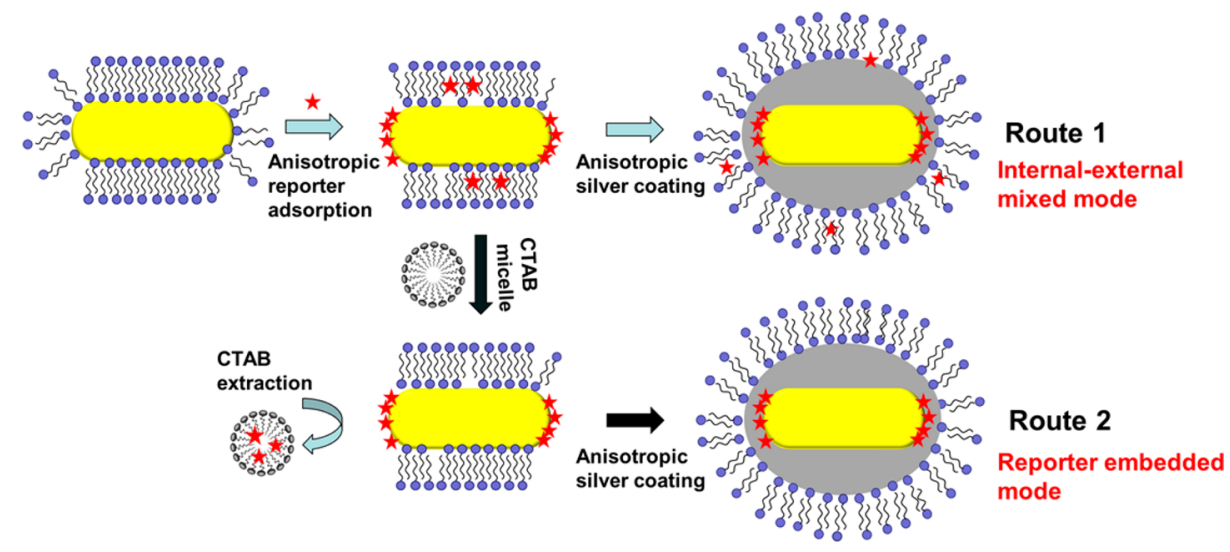

Figure 1. Schematic illustration of synthesis procedures for "internal-external" mixed tags and Raman reporter-embedded SERS tags.
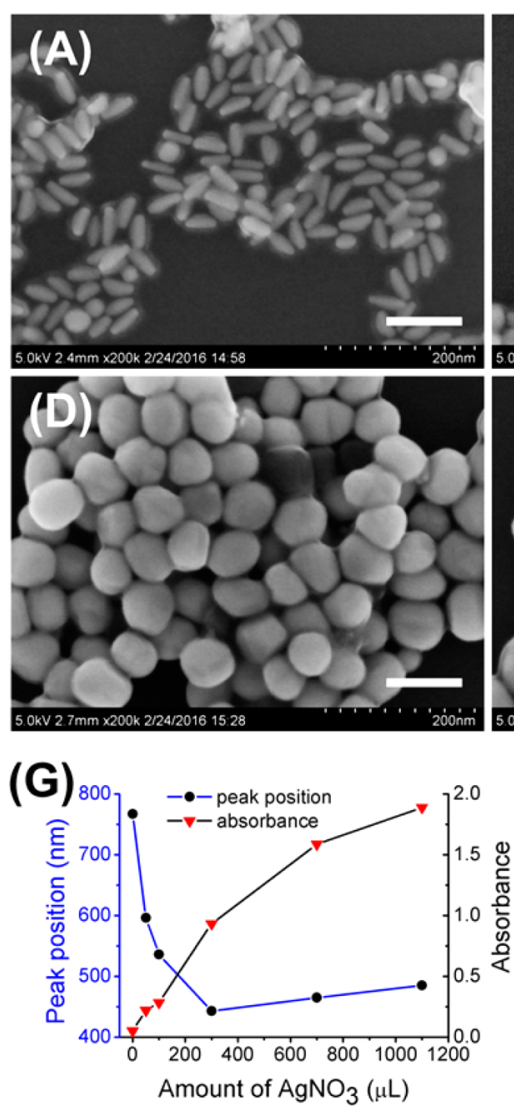
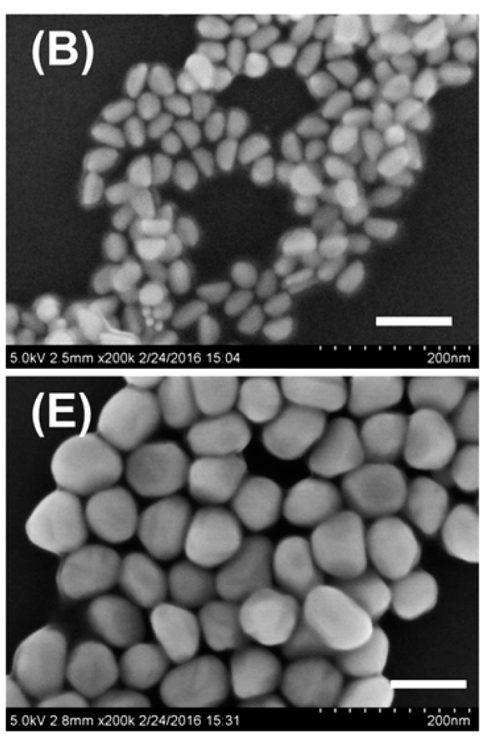

(H)

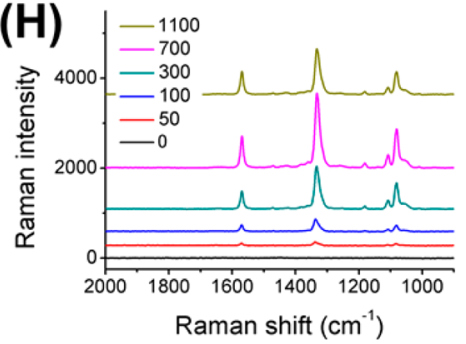

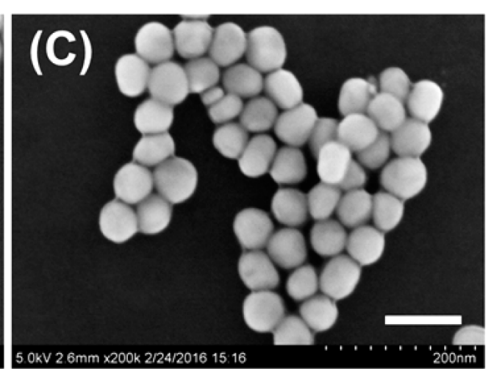

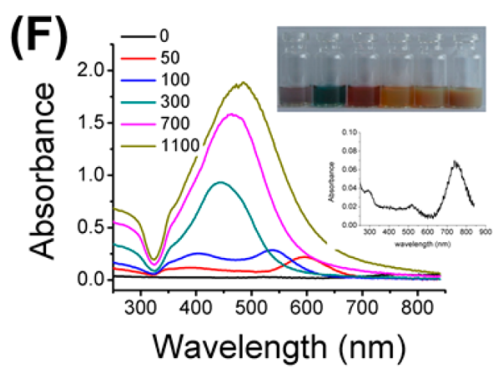

(l)

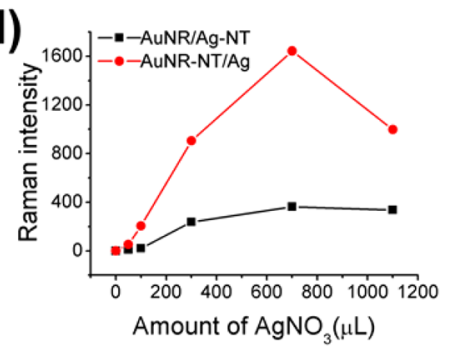

Figure 2. SEM images of AuNR/Ag "internal-external" mixed tags with different shapes synthesized using different amounts of $\mathrm{AgNO} \mathrm{O}_{3}$ : (A) $50 \mu \mathrm{L}$, (B) $100 \mu \mathrm{L}$, (C) $300 \mu \mathrm{L},(D) 700 \mu \mathrm{L}$, (E) $1100 \mu \mathrm{L}$. Scale bar: $100 \mathrm{~nm}$. (F) Photographs of aqueous dispersions of tag solution prepared with different volumes of $\mathrm{AgNO}_{3}$ solution (50, 100, 300, 700, and $1100 \mu \mathrm{L}$ from left to right), and the corresponding UV-vis-NIR absorption spectra. The inset shows the expanded view of the UV-vis-NIR absorption spectrum of AuNR seed. (G) Plots of the absorption peak position and absorbance intensity of the growth products as a function of the volume of $\mathrm{AgNO}_{3}$ solution. (H) SERS spectra of as-prepared tags, and (I) Plots of SERS intensity of the as-prepared tags and conventional "external" tags as a function of the volume of $\mathrm{AgNO}_{3}$ solution for synthesis of corresponding NPs. The Raman intensities refer to the Raman peak at $1330 \mathrm{~cm}^{-1}$.

of $\mathrm{HAuCl}_{4}$ solution $(0.5 \mathrm{mM}, 2 \mathrm{~mL})$ using a syringe pump at an injection rate of $2 \mathrm{~mL} / \mathrm{h}$. The reaction was allowed to proceed at 27 ${ }^{\circ} \mathrm{C}$ for $10 \mathrm{~min}$ after the injection had been finished. The product was AuNS with a diameter of $55 \mathrm{~nm}$. One milliliter of the as-prepared AuNS (16 or $55 \mathrm{~nm}$ ) colloid was centrifuged twice at the speed of $9000 \mathrm{rpm}$ for $10 \mathrm{~min}$ and redispersed in $950 \mu \mathrm{L}$ of water. Then, Raman reporter (NT) incubation, washing with $\mathrm{CTAB}$ solution, and the silver shell coating process were performed in a similar manner as that described for the preparation of AuNR seeded RE tags.
Preparation of Silver NPs. Silver NPs were used as SERS substrates to detect the Raman reporters extracted by CTAB solution, which were prepared by the reduction of $\mathrm{AgNO}_{3}$ with $\mathrm{NH}_{2} \mathrm{OH} \cdot \mathrm{HCl}$ at room temperature. ${ }^{30}$ Briefly, $10.44 \mathrm{mg}$ of $\mathrm{NH}_{2} \mathrm{OH} \cdot \mathrm{HCl}$ was dissolved in $89 \mathrm{~mL}$ of water, followed by the addition of $\mathrm{NaOH}(0.3 \mathrm{M}, 1 \mathrm{~mL})$ to maintain an initial alkaline $\mathrm{pH}$. Then, $10 \mathrm{~mL}$ of $10 \mathrm{mM} \mathrm{AgNO}_{3}$ solution was added to the above solution while stirring. The reaction mixture was stirred for $1 \mathrm{~h}$.

Tag Aggregation Tests. To explore the NP aggregation effect on the SERS signals of "internal-external" mixed tag and RE tag, the tag 

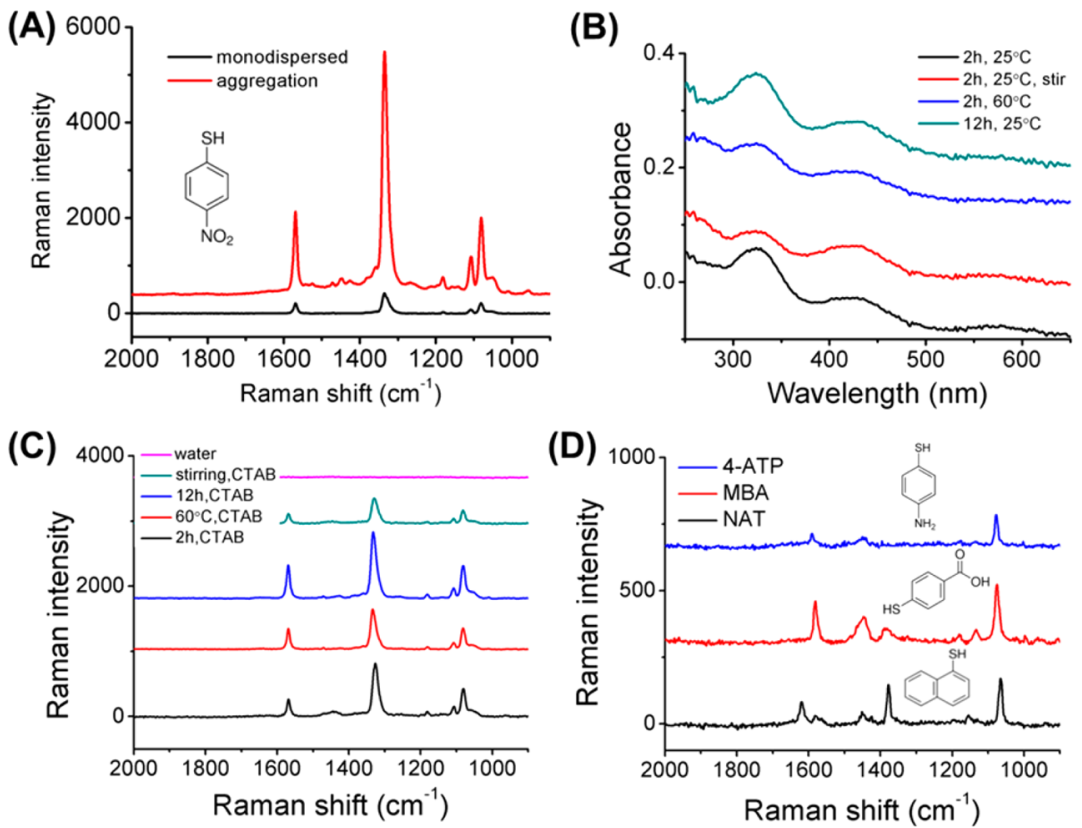

Figure 3. (A) SERS spectra of "internal-external" mixed tags before and after mild aggregation. (B) UV-vis absorption spectra of CTAB washing supernatant of AuNR-NT complex obtained from different incubation conditions. (C) SERS spectra of silver NP colloid after adding the CTAB or water washing supernatant from different incubation conditions using NT as Raman reporter. (D) SERS spectra of silver NP colloid after adding the CTAB washing supernatant. The AuNR seed was prepared by incubation with NAT, MBA, or 4-ATP as a Raman reporter, respectively.

aggregation was induced in a controllable manner. Briefly, $200 \mu \mathrm{L}$ of ethanol was added into $200 \mu \mathrm{L}$ of tag solution; the color of the solution changed from yellow to brown immediately, which indicated the aggregation of the tags. Then, $50 \mu \mathrm{L}$ of PVP $(20 \mathrm{~g} / \mathrm{L})$ was added to the mixture immediately to quench the aggregation process and stabilize the NP to some extent. ${ }^{31}$ All SERS signal measurements were performed at once after the addition of PVP solution to obtain the initial signal of each sample.

Test on the pH-Sensitive Property of MBA Embedded Tags. Small pieces of filter paper were dipped into the tag solution for the tag adsorption. Then, the papers loaded with tags were placed in acetate buffer ( $\mathrm{pH} \mathrm{4}$ ) and phosphate buffer $(\mathrm{pH} 8)$ solutions, followed by immediate SERS measurements, to avoid the dissolution of the silver shell of the tags.

Cell Culture. SMMC7721 (a liver cancer cell line) cells were grown as a monolayer in a humidified incubator at $37^{\circ} \mathrm{C}$, in air $/ \mathrm{CO}_{2}$ (95:5) and in an RPMI-1640 medium that was supplemented with $10 \%$ fetal bovine serum. For all experiments, cells were harvested by using trypsin and were resuspended in fresh medium before plating.

Cell Imaging. SMMC7721 cells $\left(2 \times 10^{4}\right)$ were seeded onto glass coverslips in a 24-well plate with $500 \mu \mathrm{L}$ of culture medium; the cells were cultured for $12 \mathrm{~h}$ to allow the cells to attach. Then, $100 \mu \mathrm{L}$ of each tag was added into the cells. After incubation for 2, 4, 8, 10, $24 \mathrm{~h}$, the cell monolayer on the coverslip was observed with Raman microscopy and the SERS signals were measured at the same time.

\section{RESULTS AND DISCUSSION}

Preparation of "Internal-External" Mixed Tags. As shown in Route 1 in Figure 1, AuNR-reporter seeds were first prepared by the use of a routine Raman reporter labelingwashing procedure. ${ }^{32-35}$ Briefly, NT was added as the Raman reporter to AuNR solution during vigorous stirring and the resultant mixture was allowed to react for $2 \mathrm{~h}$. The AuNR-NT complex was purified by centrifugation and washed twice with deionized water. Then, the complex was used as seed and subjected to a silver coating reaction by the reduction of $\mathrm{AgNO}_{3}$ with AA under basic conditions.
The shape and morphology of the tags evolved with the increase of $\mathrm{Ag}^{+}$concentration in the growth solution. As shown in Figure $2 \mathrm{~A}$, at a lower volume of $\mathrm{AgNO}_{3}(50 \mu \mathrm{L})$, the tag showed a cylindrical rod shape similar to that of AuNR (Figure $\mathrm{S} 1 \mathrm{~A}$ ) and the $\mathrm{Ag}$ shell was homogeneous over the AuNR. When the volume of $\mathrm{AgNO}_{3}$ was increased to $100 \mu \mathrm{L}$, an anisotropic Ag coating occurred, leading to the tags with an orange slice-like shape (Figure 2B). This anisotropic growth feature was consistent with $\mathrm{Au} / \mathrm{Ag} \mathrm{NR}$ growth from bare $\mathrm{AuNR},{ }^{27,36}$ which indicated that the adsorbed Raman reporters did not influence the growth behavior of the silver shells. When the volume was increased to more than $300 \mu \mathrm{L}$, the tags became spherical and their size increased (Figure 2C-E).

Figure $2 \mathrm{~F}$ shows a series of color changes for the NP solutions from pink for the AuNR seeds to green, orange, and finally yellow for the AuNR/Ag tags. The corresponding UVvis absorption spectra indicated that the longitudinal SPR band blue-shifted as a result of the decrease in the aspect ratio of AuNR, and a new peak appeared at $380 \mathrm{~nm}$ that originated from the transverse dipole resonance of electrons on the thick side of the Ag shell. With increasing amounts of $\mathrm{AgNO}_{3}$, the absorbance of the solution increased from 0.05 to 1.9 according to the main peak (Figure 2G). The SERS spectra of the samples are shown in Figure $2 \mathrm{H}$. When the volume of $\mathrm{AgNO}_{3}$ was increased from 50 to $700 \mu \mathrm{L}$, the SERS intensity of the tags increased continuously and reached a maximum at $700 \mu \mathrm{L}$, which was approximately 460 times higher than the intensity of the AuNR seeds calculated from the Raman peak at $1330 \mathrm{~cm}^{-1}$ (Figure S1B). This signal enhancement is attributed to $\mathrm{Ag}$ having a much greater Raman enhancement ability than $\mathrm{Au}^{37}$ When more $\mathrm{AgNO}_{3}$ was added, a decrease in Raman intensity was observed because the light-shadowing effect of the Ag shell became dominant. The Ag shell with increasing thickness made it more difficult for the incident laser to reach the reporters and the scattering signal to escape. ${ }^{5}$ Therefore, the combination of these two effects determined an optimum Ag shell thickness for 

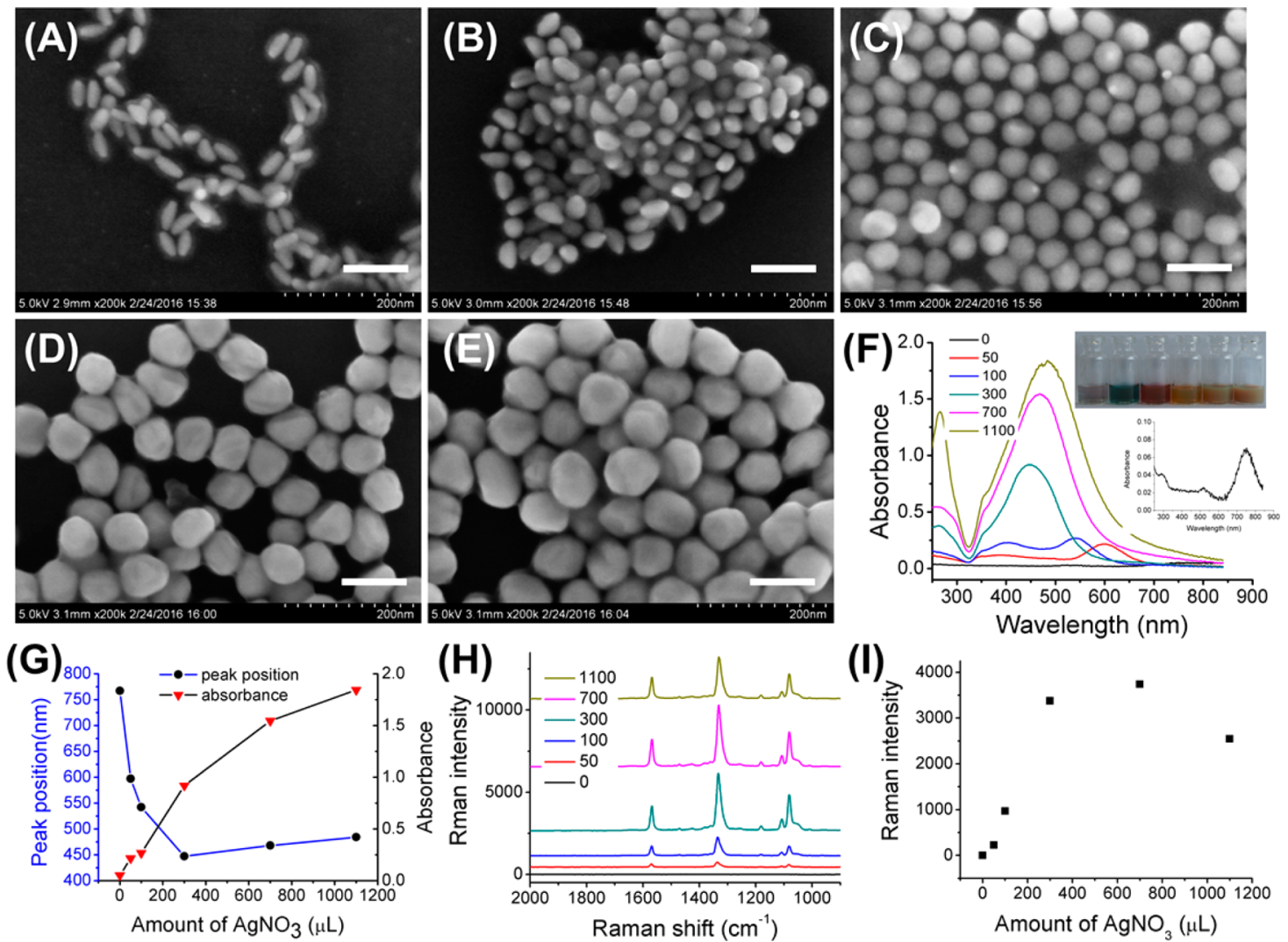

Figure 4. SEM images of AuNR-NT/Ag RE tags with different shapes synthesized using different amounts of $\mathrm{AgNO}_{3}$ : (A) $50 \mu \mathrm{L}$, (B) $100 \mu \mathrm{L}$, (C) $300 \mu \mathrm{L}$, (D) $700 \mu \mathrm{L}$, (E) $1100 \mu \mathrm{L}$. Scale bar: $100 \mathrm{~nm}$. (F) Photographs of aqueous dispersions of tags prepared with different volumes of AgNO solution (50,100, 300, 700, and $1100 \mu \mathrm{L}$ from left to right), and the corresponding UV-vis-NIR absorption spectra. The inset shows the expanded view of the UV-vis-NIR absorption spectrum of AuNR seed. (G) Plots of the absorption peak position and absorbance intensity of the growth products as a function of the volume of $\mathrm{AgNO}_{3}$ solution. (H) SERS spectra of as-prepared tags, and (I) plots of SERS intensity as a function of the volume of $\mathrm{AgNO}_{3}$ solution. The Raman intensities refer to the Raman peak at $1330 \mathrm{~cm}^{-1}$.

RE tags to obtain the best sensitivity. By contrast, for conventional "external" SERS tags in which Raman reporters were attached on the surface of AuNR/Ag NP substrates (Figure S2), a nearly continuously increasing SERS intensity was observed with increasing silver content of AuNR/Ag NPs, and the SERS intensity from NPs of the same silver content was much lower (Figure 2I).

Furthermore, an aggregation test was performed to verify the $\mathrm{RE}$ structure of the tags. It is well-known that the aggregation of "external" SERS tags will result in a dramatic signal increase because Raman reporters located at the junctions of the NP aggregates (i.e., hotspots) feel much enhanced electromagnetic fields. However, the signal for the RE tags was inert due to the insulation of reporters and hotspots by the outer metal shell. For instance, Chen's group reported that the signal of AuMBA/Ag ( $5 \mathrm{~nm}$ shell) RE tags barely changed after NaClinduced tag aggregation. ${ }^{5}$ In our case, the aggregates of the tags ( $15 \mathrm{~nm} \mathrm{Ag}$ shell on the side of the AuNR) were prepared with the addition of ethanol and PVP solution to the tag solution (Figure S3). Strangely, the signal intensity of the tags increased significantly to approximately 5000 counts after aggregation (Figure 3A). Considering that the shell of the tags was thick enough for reporter isolation, it could be deduced that not all reporters were embedded inside metals and there must be a portion of NT molecules attached on the outer surface of the $\mathrm{Ag}$ shell. These molecules were incorporated in the hotspots of the aggregates and led to the signal enhancement. To emphasize the different locations of the reporters, this tag was defined as an "internal-external" mixed tag.

Investigation of Physically Adsorbed Raman Reporters on AuNR. We speculated that the formation of "internalexternal" mixed tags originated from the different locations of Raman reporters on the AuNR-reporter seeds. After mixing with AuNR colloid, a majority of Raman reporters adsorbed on AuNR via strong $\mathrm{Au}-\mathrm{S}$ bonds and could be embedded inside the Ag shell, which were considered as "effective" reporters. Meanwhile, there should be a certain number of reporters intercalated in the CTAB bilayers of the AuNR via hydrophobic interactions between the alkyl chain of CTAB and the benzene rings of the Raman reporters. ${ }^{38}$ These "ineffective" reporters could not embed at the $\mathrm{Au}-\mathrm{Ag}$ interface but located on the outer surface of the resultant tags after Ag shell coating.

To verify this speculation, $0.2 \mathrm{M} \mathrm{CTAB}$ solution was applied for washing the AuNR-reporter complex because of its much stronger ability than water for the extraction of the physically adsorbed reporters, as illustrated in Route 2 (Figure 1). CTAB molecule exchange occurred between AuNR seeds and large excess of CTAB micelles in the washing solution. The physically adsorbed NT on AuNR might transfer to CTAB micelles and could be discarded after centrifugation, whereas this washing process would not influence the chemically adsorbed NT molecules on the AuNR seeds. The UV-vis spectra of NT were measured to monitor this extraction capability. AuNR was incubated with $10^{-4} \mathrm{M} \mathrm{NT}$, centrifuged, followed by a washing step with CTAB solution or pure water. 

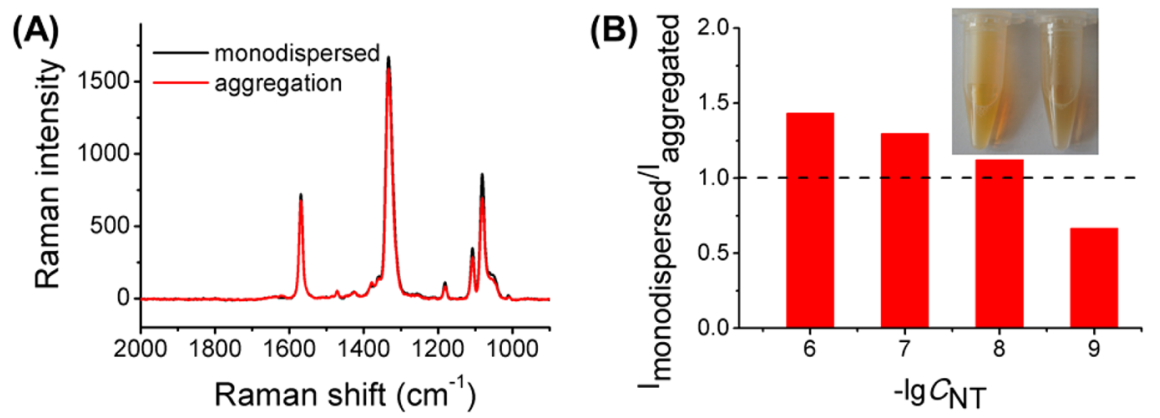

Figure 5. (A) SERS spectra of AuNR-NT/Ag(700) RE tags before and after aggregation. (B) SERS intensity ratio of RE tag solution with different concentrations of additional NT before and after aggregation. Inset shows photographs of RE tag solution before (left) and after (right) aggregation.

The washing supernatants were collected for comparison. As shown in Figure S4, a weak NT absorbance could be detected in the water supernatant. In contrast, a strong NT absorbance appeared from CTAB washing supernatant, which indicated that a large amount of NT was released from AuNR in the extraction step. When a lower NT concentration was used $(5 \times$ $\left.10^{-5} \mathrm{M}\right)$, the characteristic absorption bands were also measured (Figure 3B). It was estimated that approximately 4 $\times 10^{-6} \mathrm{M}$ of the NT (i.e., $8 \%$ of the NT molecules) was physically adsorbed on the AuNR seeds according to the UVvis linear curve of $\mathrm{NT}$ in $0.2 \mathrm{M} \mathrm{CTAB}$, and the number of chemically adsorbed NT on each AuNR was approximately 1.9 $\times 10^{4}$ (Supporting Information).

This coexisting chemical and physical adsorption of Raman reporters on AuNR is a universal phenomenon. Multiple AuNR-reporter incubation conditions, which included vigorous stirring, prolonging the incubation time, or increasing the incubation temperature, illustrated similar conclusions according to the NT detection results by UV-vis spectra (Figure 3B) and more sensitive SERS measurements. After the addition of the CTAB washing supernatant to the silver NP colloids, strong characteristic Raman peaks of NT appeared (Figure 3C). This universal feature was further verified by using other thiolcontaining Raman reporter molecules such as MBA, NAT, and 4-ATP. After the addition of each CTAB washing supernatant to Ag colloid, the corresponding Raman spectrum could be detected (Figure 3D).

This is an important result that contradicts traditional beliefs. Typically, it is considered that all attached thiol reporters on AuNR or AuNR/Ag NPs are stabilized via strong $\mathrm{Au}(\mathrm{Ag})-\mathrm{S}$ bonds. Therefore, the SERS tags are usually prepared by simply mixing Raman reporters and NPs and then centrifuging and washing the mixture with water to remove excess reporters. $^{32-35}$ Our results revealed that the CTAB bilayers of AuNRs were highly stable, ${ }^{19,39}$ and there were always reporters intercalated in the $\mathrm{CTAB}$ bilayers, even for small thiolcontaining molecules that bind strongly with metals and have relatively small hydrophobic interactions with the alkyl chains of CTAB.

The above results hint at two problems that have previously been ignored when investigating the SERS features of NPs with CTAB ligands: (1) A missed factor in the evaluation of the SERS enhancement ability. For example, the distinction of SERS enhancement ability of AuNRs with different aspect ratios was solely explained by the difference in aspect ratiodependent electromagnetic enhancement. However, the chemical and physical adsorption ratio of Raman reporters was also influenced by the aspect ratio, as the AuNRs with larger aspect ratios had denser CTAB bilayers on the sides and might hold more "ineffective" reporters. ${ }^{34}$ (2) Physically adsorbed reporters might affect the signal stability of SERS tags. These reporters were easily disturbed or released from the tags and readsorbed via the $\mathrm{Au}-\mathrm{S}$ bond as "effective" reporters, which induced interference with the original signal. These problems require special attention in future SERS tag studies.

Preparation of RE SERS Tags. Purified seeds were prepared by washing the AuNR-NT complex with a $0.2 \mathrm{M}$ $\mathrm{CTAB}$ solution. Four rounds of washing could completely clear the physically adsorbed NT molecules (Figure S5). After the washing process, the monodispersity of the seeds could still be maintained (Figure S6). Subsequently, RE tags were prepared by coating the seeds with a Ag layer. As shown in Figure 4A-E, anisotropic growth behavior of the NPs was observed, as indicated by the faster growth of the Ag shell on the side of the AuNR than on the tip of the AuNR (Figure S7A). When the $\mathrm{Ag}^{+}$volume was increased to $300 \mu \mathrm{L}$, the AuNR, including both tips, was completely encapsulated by silver, as shown by the contrast in the TEM images (Figure S8). The color and extinction spectra of the NPs showed the same evolution in properties as the spectra obtained from the "internal-external" mixed tags (Figure 4F,G), and the monodispersity of the tags was satisfactory (Figure S7B). Moreover, the SERS signal variation also supported a similar "rise-fall" principle and the intensity was as strong as 3700 counts at its maximum. (Figure 4H,I).

As shown in Figure 5A, the signal of the tags was nearly stable after ethanol-induced aggregation, indicating the successful formation of the RE structure. The amount of NT that adsorbed on the outer shell of the RE tag was further roughly estimated by adding known concentrations of NT reporters to the RE tag samples, followed by the addition of ethanol to form similar aggregation states. As shown in Figure $5 B$, the SERS signal of aggregated NPs at $1330 \mathrm{~cm}^{-1}$ increased by $31 \%$ and $12 \%$ when the added NT concentrations were $10^{-7}$ and $10^{-8} \mathrm{M}$, respectively. When the added NT concentration was $10^{-9} \mathrm{M}$, the aggregation resulted in a decreased signal. The results indicated that most of the attached NT were embedded and the concentration of NT adsorbed on the tag surface was less than $10^{-8} \mathrm{M}$.

We speculated that the high sensitivity of the AuNR seeded $\mathrm{RE}$ tag was attributed to the tip-selective Raman reporter adsorption and the anisotropic silver coating properties of AuNRs. To prove this idea, two types of RE tags with controlled structures, i.e., "mPEG on AuNR Tip" RE tags and gold nanosphere (AuNS) seeded RE tags, were prepared for comparison. 

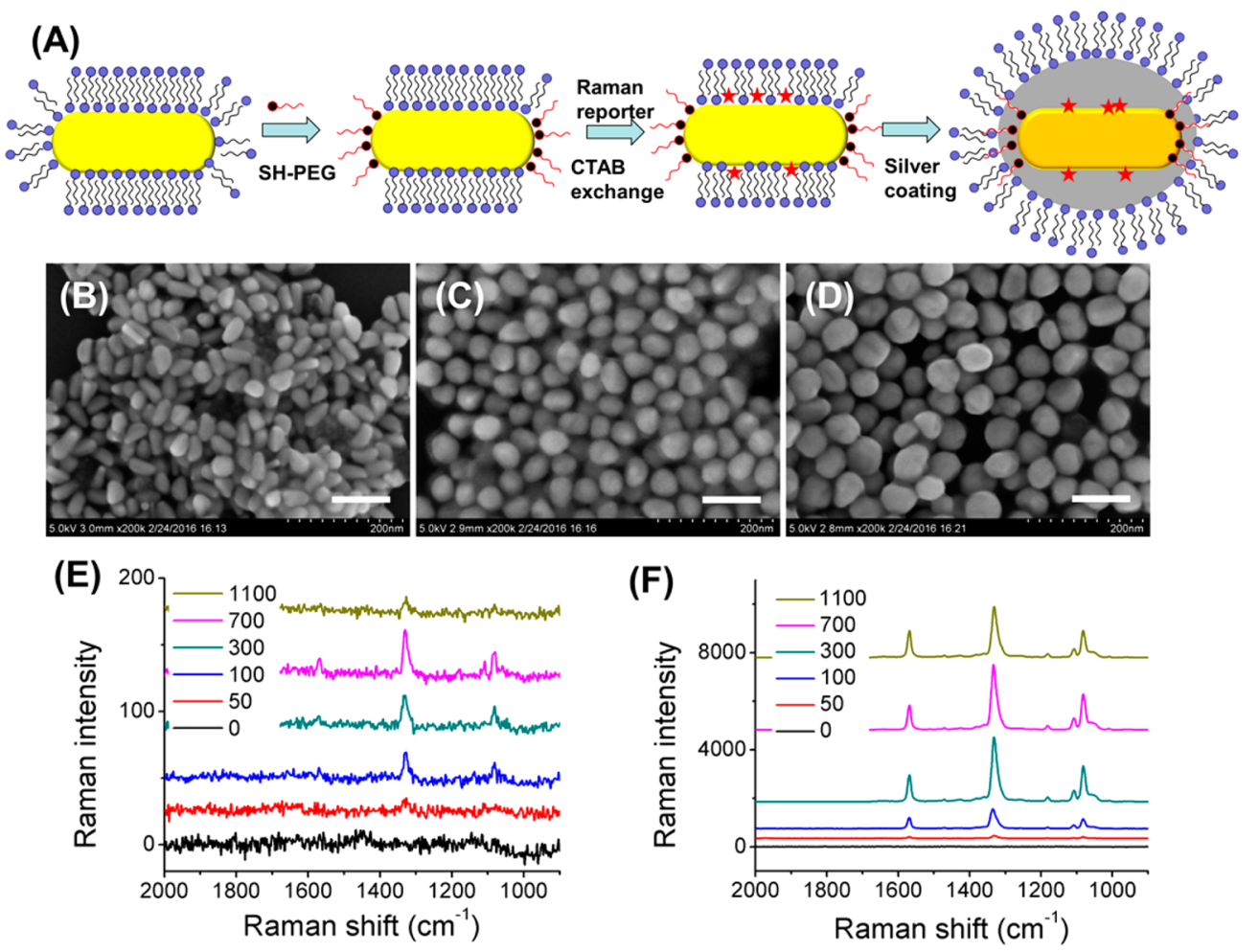

Figure 6. (A) Synthesis procedure of "mPEG on tip" RE tags. SEM images of "mPEG on tip" RE tags prepared with addition of (B) 100, (C) 300, and (D) $700 \mu \mathrm{L}$ of $\mathrm{AgNO}_{3}$. Scale bar: $100 \mathrm{~nm}$. SERS spectra of as-prepared "mPEG on tip" RE tags (E) and RE tags without mPEG treatment (F) prepared from different amounts of $\mathrm{AgNO}_{3}$ solution. The encapsulated NT was estimated to be approximately $4 \times 10^{-5} \mathrm{M}$ in both tags.

SERS Property of the "mPEG on AUNR Tip" RE SERS

Tags. The anisotropic reporter adsorption feature of AuNR would be advantageous to achieve sensitivity. On the one hand, it had been reported that an enhanced electric field exists at the tip of an AuNR, which was mainly responsible for the SERS enhancement ability. ${ }^{21}$ Simultaneously, thiol-containing Raman reporters preferentially attached on the tips of AuNR because of the low capping density of CTAB at these locations. This denoted a perfect match as more reporters appear at the hotspots in the AuNR seed, which might be favorable for the acquirement of strong SERS signals from the RE tags. To verify this speculation, the "mPEG on AuNR tip" RE tags were prepared as a controlled sample by using the seed NP with Raman reporters located at the sides of the AuNRs, as illustrated in Figure 6A. For AuNR washed twice with water, the tips of the AuNR were almost bare and the sides of the AuNR were still covered by the CTAB bilayer. The added mPEG-SH to the AuNR colloid was prior to occupy the bare $\mathrm{Au}$ surface at the tips, and it was hard for them to attach on the side of the AuNR due to the protection of the CTAB bilayer. In the following Raman reporter incubation step, most reporters were forced to attach to the sides of the AuNRs. Figure 6B-D shows the formation of the core-shell RE structure from the "mPEG on AuNR Tip" seed. Due to the additional coverage effects of mPEG-SH, the number of attached reporters decreased slightly by nearly $11.6 \%$, while the SERS intensity of the RE tags after silver coating decreased sharply to $1 / 125$ of the intensity of typical RE tags with the same number of embedded reporters (Figure 6E,F).

SERS Property of AuNS Seeded Tags. Anisotropic silver coating properties on AuNRs was also beneficial for obtaining strong SERS signal. The fast silver growth on the sides of AuNRs ensured a considerable silver content of the tags for
SERS enhancement. The thin shells on the tips prevented shadowing the reporters adsorbed at the tip "hotspot" too much. For comparison, conventional gold NSs were used as seeds for the preparation of AuNS-NT/Ag tags, in which the silver shells were homogeneously coated on AuNSs. Two sizes (16 and $55 \mathrm{~nm}$ ) were tested, which were comparable to the transverse and longitudinal sizes of the AuNR, respectively. When $16 \mathrm{~nm}$ AuNSs were used as seeds, the signal increased significantly with the diameter of tags increasing to about 40 $\mathrm{nm}$ upon silver coating. The maximum intensity was about 60 times higher than the intensity from the seeds (Figure S9). This value was lower than that from AuNR-seeded tags with similar silver shell thickness at the tip. For $55 \mathrm{~nm}$ AuNS seed, no significant signal increase was detected during the NP growth. The maximum Raman intensity of the tags was only 3.3 times higher than that of AuNS seeds (Figure S10).

Verifying the Raman Reporter Encapsulation at AuNR Seed Tips. One perplexing problem introduced by the sidepreferring anisotropic growth feature of AuNRs was whether the tip of the AuNR seed was fully coated by the silver shell. The shells were too thin to be clearly observed by SEM or TEM, especially at lower $\mathrm{AgNO}_{3}$ concentrations. To address this problem, a novel verification method was proposed which applied the pH-sensitive SERS property of the MBA Raman reporter. The Raman peak of MBA at $1430 \mathrm{~cm}^{-1}$ (assigned to the $\mathrm{COO}^{-}$-stretching mode) was sensitive to the dissociation of the carboxyl group. The signal ratio of the peaks at $1430 \mathrm{~cm}^{-1}$ to the stable aromatic ring vibration at $1076 \mathrm{~cm}^{-1}$ could be used to sense $\mathrm{pH}$ variation. ${ }^{40,41}$ We speculated that, if MBA was embedded inside metal layers, this $\mathrm{pH}$-sensitive feature would vanish due to the insulation of MBA from the tag's local environments. With this in mind, AuNR-MBA/Ag RE tags (Figure S11) and AuNR/Ag-MBA "external" tags were 

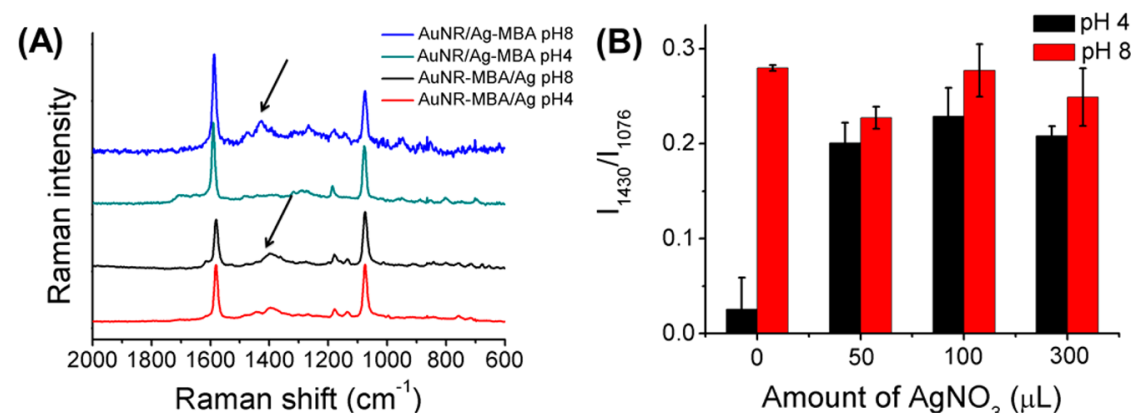

Figure 7. (A) SERS spectra response of AuNR-MBA/Ag(700) RE tags and AuNR/Ag(700)-MBA "external" tags at pH 4 and $\mathrm{pH} 8$ conditions. For "external" tags, the $\mathrm{COO}^{-}$-stretching mode (indicated by arrows) disappeared in acidic medium, while it remained for RE tags. (B) The peak ratio of 1430 and $1076 \mathrm{~cm}^{-1}$ at $\mathrm{pH} 4$ and $\mathrm{pH} 8$ conditions for "external" tags and $\mathrm{RE}$ tags prepared with different amounts of $\mathrm{AgNO}_{3}$.
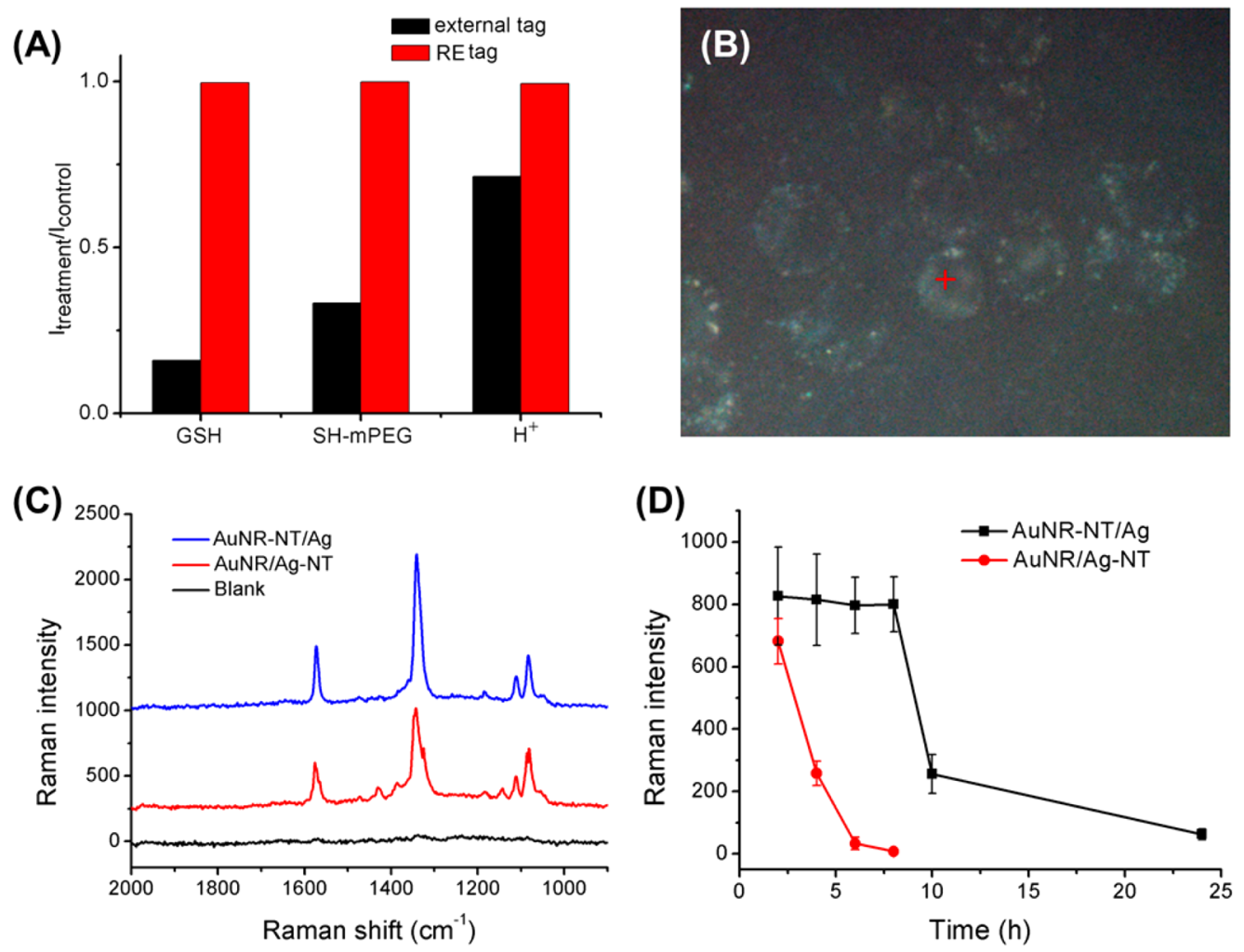

Figure 8. (A) Signal stability comparison of "external" and RE tags in different stimulus conditions. The concentration of glutathione and mPEG-SH was $10^{-5} \mathrm{M}$, and SERS measurements were performed after mixing for $30 \mathrm{~min}$. The low $\mathrm{pH}$ test was conducted by adding $100 \mu \mathrm{L}$ of $\mathrm{HCl}(0.1 \mathrm{M})$ to $1 \mathrm{~mL}$ of these two tag solutions, followed by SERS measurements after $90 \mathrm{~min}$. (B) Dark-field image of SMMC7721 cells after incubating RE tags for 2 h. (C) Representative Raman spectra acquired from "external” and RE SERS tag labeled SMMC7721 cells. (D) Plot showing the Raman intensity of both tags in cell for different incubation durations. The Raman intensities refer to the Raman peak at $1330 \mathrm{~cm}^{-1}$.

synthesized and loaded on test papers for SERS spectra measurements under different $\mathrm{pH}$ conditions. As illustrated in Figure 7A, for "external" tags, the Raman peak at $1430 \mathrm{~cm}^{-1}$ decreased dramatically after changing the $\mathrm{pH}$ from 8 to 4 . By contrast, the Raman spectrum profile of the AuNR-MBA/ $\mathrm{Ag}(700) \mathrm{RE}$ tag was irrelevant to the $\mathrm{pH}$ change because of the protection of the Ag shell. The RE tag of a thinner silver shell was also tested. As shown in Figure 7B, even for the AuNRMBA/Ag(50) RE tag with a very thin Ag shell at the AuNR tip, the peak ratio of 1430 and $1076 \mathrm{~cm}^{-1}$ was nearly stable after the $\mathrm{pH}$ changes, which implied the successful encapsulation of the reporters. By contrast, the peak ratio for the "external" tags decreased sharply from 0.28 to 0.025 . The proposed $\mathrm{pH}$ sensitive SERS peak test was effective for the confirmation of thin metal shell coverage and provided a complementary method for previously applied TEM and corresponding EDS line scan analyses. The preparation of RE tags was also done using NAT as the Raman reporter, which proved that the synthesis could be expanded to other reporters (Figure S12).

Signal Stability of RE SERS Tags. Ag NP-based SERS tags suffer from poor signal stability due to the chemical activity of Ag NPs with many biological and environmental factors, such as thiol-molecules, $\mathrm{O}_{2}, \mathrm{H}^{+}$, etc. ${ }^{42-46}$ As shown in Figure $8 \mathrm{~A}$, the signal of the conventional "external" tags was disturbed easily when the tags were incubated with glutathione, mPEG-SH, or in acidic medium because the dissolution of only a single layer of $\mathrm{Ag}$ atoms could result in the serious release of Raman reporters from Ag NPs. By contrast, the signal of the RE tag was not determined by the "first atom layer" effect but by the 
thickness of the entire silver layer, which was more robust in these conditions.

Furthermore, the intracellular SERS signal response of both tags was investigated in living SMMC7721 cells. During cell incubation, NPs passed through the cytomembrane and were trapped in the endosome, encountering endogenous small thiol molecules and an acidic environment with a $\mathrm{pH}$ of approximately 5, which could promote the dissolution of silver. $^{47}$ It was interesting to check the signal stability distinctions of "external" tags and RE tags in these cells. From the dark-field optical imaging of live cells, it was observed that the tags were distributed in cytoplasmic regions after $2 \mathrm{~h}$ of incubation, which indicated the uptake of NPs (Figure 8B). Simultaneously, SERS measurements were performed using a $632.8 \mathrm{~nm}$ laser with a power of $\sim 1 \mathrm{~mW}$. Figure $8 \mathrm{C}$ shows that strong and characteristic SERS signals of NT of both tags were detected from the single cell at $2 \mathrm{~h}$. However, the prolonged time-dependent signal feature was different for the two tags. As shown in Figure 8D, the SERS intensity of the "external" tags underwent a continuous, sharp decrease upon cell incubation. The signal totally disappeared after $8 \mathrm{~h}$. By contrast, the signal of the RE tags was pretty stable in the first $8 \mathrm{~h}$, followed by a dramatic decrease in the subsequent $16 \mathrm{~h}$ incubation period. This result implied that the silver shell could provide protection at the initial stage, but this function disappeared as a result of the gradual silver dissolution and the exposure of the Raman reporters to the surroundings.

\section{CONCLUSIONS}

We have prepared and investigated the signal properties of novel RE SERS tags with Raman reporters embedded between the AuNR and Ag shell. We found that physically and chemically adsorbed reporters always coexist on AuNR seeds due to the strong capacity of CTAB bilayers to hold molecules, which complicated the synthesis of pure RE structures compared with the use of AuNP seeding and capping by small citrate ligands. Moreover, the hotspot effect at the tip of AuNRs remained and was even amplified in the RE tags. Raman reporters adsorbed at the tips provided a dominant contribution to the SERS intensity, which rendered a tag with high sensitivity. The anisotropic growth of the silver shell that occurred during RE preparation also benefited signal enhancement. Moreover, a novel method was proposed to verify the thin silver layer coverage by monitoring the Raman intensity variation of a $\mathrm{pH}$-sensitive peak of the $\mathrm{MBA}$ reporter in different $\mathrm{pH}$ conditions. Taking advantage of the silver shell protection, RE tags showed better in vitro and in vivo signal stability compared with their external labeling counterparts. We believe that these sensitive and stable RE tags prepared relying on the anisotropic features of AuNR can draw broad interests and become useful tools in molecular detection and bioimaging applications.

\section{ASSOCIATED CONTENT}

\section{S Supporting Information}

The Supporting Information is available free of charge on the ACS Publications website at DOI: 10.1021/acsami.6b04216.

SERS spectrum of NT-labeled AuNR seed, SEM images of AuNR/Ag NPs, UV-vis spectra of supernatant after washing the AuNR-NT complex with water and 0.2 M CTAB solution, UV-vis spectra of AuNR after four rounds of $C T A B$ washing, SERS spectra of the mixture of
Ag colloid and CTAB washing supernatants, quantification of attached Raman reporters on each AuNR, dynamic light scattering analysis result of the tags, morphologies and SERS properties of AuNS based tags, plots of the lengths and diameters of the growth tags as a function of the volume of the added $\mathrm{AgNO}_{3}$ solution, and morphological and optical properties of AuNRMBA/Ag and AuNR-NAT/Ag RE tags (PDF)

\section{AUTHOR INFORMATION}

\section{Corresponding Authors}

*E-mail: yqwang@yic.ac.cn. Fax: +86 535 2109130. Tel: +86 5352109130 (Y.W.).

*E-mail: sunkx@ytu.edu.cn. Fax: +86 535 6706066. Tel: +86 5353808266 (K.S.).

*E-mail: lxchen@yic.ac.cn. Fax: +86 535 2109130. Tel: +86 5352109130 (L.C.).

\section{Notes}

The authors declare no competing financial interest.

\section{ACKNOWLEDGMENTS}

Financial support from the National Natural Science Foundation of China (81573393, 21305157, 81102415, and 21575159), The 12th Five Years Key Programs for Science and Technology Development of China (2013ZX09402201), the Natural Science Foundation of Shandong (ZR2014HM062 and ZR2013HQ009), and the Science and Technology Development Plan of Shandong Province of China (2014GGF01074) is gratefully acknowledged.

\section{REFERENCES}

(1) Wang, Y.; Yan, B.; Chen, L. SERS Tags: Novel Optical Nanoprobes for Bioanalysis. Chem. Rev. 2013, 113, 1391-1428.

(2) Lane, L. A.; Qian, X.; Nie, S. SERS Nanoparticles in Medicine: From Label-Free Detection to Spectroscopic Tagging. Chem. Rev. 2015, 115, 10489-10529.

(3) Abramczyk, H.; Brozek-Pluska, B. Raman Imaging in Biochemical and Biomedical Applications. Diagnosis and Treatment of Breast Cancer. Chem. Rev. 2013, 113, 5766-5781.

(4) Gandra, N.; Singamaneni, S. Bilayered Raman-Intense Gold Nanostructures with Hidden Tags (BRIGHT) for High-Resolution Bioimaging. Adv. Mater. 2013, 25, 1022-1027.

(5) Feng, Y.; Wang, Y.; Wang, H.; Chen, T.; Tay, Y. Y.; Yao, L.; Yan, Q.; Li, S.; Chen, H. Engineering "Hot" Nanoparticles for SurfaceEnhanced Raman Scattering by Embedding Reporter Molecules in Metal Layers. Small 2012, 8, 246-251.

(6) Zhou, Y.; Lee, C.; Zhang, J.; Zhang, P. Engineering Versatile SERS-Active Nanoparticles by Embedding Reporters between AuCore/Ag-Shell through Layer-by-Layer Deposited Polyelectrolytes. J. Mater. Chem. C 2013, 1, 3695-3699.

(7) Lee, H.; Nam, S. H.; Jung, Y. J.; Park, S.; Kim, J.-M.; Suh, Y. D.; Lim, D.-K. DNA-Mediated Control of Au Shell Nanostructure and Controlled Intra-Nanogap for a Highly Sensitive and Broad Plasmonic Response Range. J. Mater. Chem. C 2015, 3, 10728-10733.

(8) Lim, D. K.; Jeon, K. S.; Hwang, J. H.; Kim, H.; Kwon, S.; Suh, Y. D.; Nam, J. M. Highly Uniform and Reproducible Surface-Enhanced Raman Scattering from DNA-Tailorable Nanoparticles with 1-nm Interior Gap. Nat. Nanotechnol. 2011, 6, 452-460.

(9) Zhou, Y.; Ding, R.; Joshi, P.; Zhang, P. Quantitative SurfaceEnhanced Raman Measurements with Embedded Internal Reference. Anal. Chim. Acta 2015, 874, 49-53.

(10) Shen, W.; Lin, X.; Jiang, C.; Li, C.; Lin, H.; Huang, J.; Wang, S.; Liu, G.; Yan, X.; Zhong, Q.; Ren, B. Reliable Quantitative SERS Analysis Facilitated by Core-Shell Nanoparticles with Embedded Internal Standards. Angew. Chem., Int. Ed. 2015, 54, 7308-7312. 
(11) Fales, A. M.; Vo-Dinh, T. Silver Embedded Nanostars for SERS with Internal Reference (SENSIR). J. Mater. Chem. C 2015, 3, 73197324.

(12) Lim, D. K.; Kim, I. J.; Nam, J. M. DNA-Embedded Au/Ag CoreShell Nanoparticles. Chem. Commun. 2008, 5312-5314.

(13) Zhao, B.; Shen, J.; Chen, S.; Wang, D.; Li, F.; Mathur, S.; Song, S.; Fan, C. Gold Nanostructures Encoded by Non-Fluorescent Small Molecules in PolyA-Mediated Nanogaps as Universal SERS Nanotags for Recognizing Various Bioactive Molecules. Chem. Sci. 2014, 5, 4460-4466.

(14) Oh, J. W.; Lim, D. K.; Kim, G. H.; Suh, Y. D.; Nam, J. M. Thiolated DNA-Based Chemistry and Control in the Structure and Optical Properties of Plasmonic Nanoparticles with Ultrasmall Interior Nanogap. J. Am. Chem. Soc. 2014, 136, 14052-14059.

(15) Shen, J.; Su, J.; Yan, J.; Zhao, B.; Wang, D.; Wang, S.; Li, K.; Liu, M.; He, Y.; Mathur, S.; Fan, C.; Song, S. Bimetallic Nano-Mushrooms with DNA-Mediated Interior Nanogaps for High-Efficiency SERS Signal Amplification. Nano Res. 2015, 8, 731-742.

(16) Gandra, N.; Portz, C.; Singamaneni, S. Multifunctional Plasmonic Nanorattles for Spectrum-Guided Locoregional Therapy. Adv. Mater. 2014, 26, 424-429.

(17) Lin, L.; Gu, H. C.; Ye, J. Plasmonic Multi-Shell Nanomatryoshka Particles as Highly Tunable SERS Tags with Built-in Reporters. Chem. Commun. 2015, 51, 17740-17743.

(18) Zhang, W.; Wang, Y.; Sun, X.; Wang, W.; Chen, L. Mesoporous Titania Based Yolk-Shell Nanoparticles as Multifunctional Theranostic Platforms for SERS Imaging and Chemo-Photothermal Treatment. Nanoscale 2014, 6, 14514-14522.

(19) von Maltzahn, G.; Centrone, A.; Park, J. H.; Ramanathan, R.; Sailor, M. J.; Hatton, T. A.; Bhatia, S. N. SERS-Coded Gold Nanorods as a Multifunctional Platform for Densely Multiplexed near-Infrared Imaging and Photothermal Heating. Adv. Mater. 2009, 21, 3175-3180.

(20) Polavarapu, L.; Porta, A. L.; Novikov, S. M.; Coronado-Puchau, M.; Liz-Marzan, L. M. Pen-on-Paper Approach toward the Design of Universal Surface Enhanced Raman Scattering Substrates. Small 2014, $10,3065-3071$.

(21) Gabudean, A. M.; Focsan, M.; Astilean, S. Gold Nanorods Performing as Dual-Modal Nanoprobes Via Metal-Enhanced Fluorescence (MEF) and Surface-Enhanced Raman Scattering (SERS). J. Phys. Chem. C 2012, 116, 12240-12249.

(22) Martín, A.; Wang, J. J.; Iacopino, D. Flexible SERS Active Substrates from Ordered Vertical Au Nanorod Arrays. RSC Adv. 2014, 4, 20038-20043.

(23) Abbas, A.; Tian, L.; Morrissey, J. J.; Kharasch, E. D.; Singamaneni, S. Hot Spot-Localized Artificial Antibodies for LabelFree Plasmonic Biosensing. Adv. Funct. Mater. 2013, 23, 1789-1797.

(24) Chen, T.; Du, C. L.; Tan, L. H.; Shen, Z. X.; Chen, H. Y. SiteSelective Localization of Analytes on Gold Nanorod Surface for Investigating Field Enhancement Distribution in Surface-Enhanced Raman Scattering. Nanoscale 2011, 3, 1575-1581.

(25) Kumar, J.; Thomas, K. G. Surface-Enhanced Raman Spectroscopy: Investigations at the Nanorod Edges and Dimer Junctions. J. Phys. Chem. Lett. 2011, 2, 610-615.

(26) Lee, S.; Anderson, L. J. E.; Payne, C. M.; Hafner, J. H. Structural Transition in the Surfactant Layer That Surrounds Gold Nanorods as Observed by Analytical Surface-Enhanced Raman Spectroscopy. Langmuir 2011, 27, 14748-14756.

(27) Lin, M.; Wang, Y.; Sun, X.; Wang, W.; Chen, L. "Elastic" Property of Mesoporous Silica Shell: For Dynamic Surface Enhanced Raman Scattering Ability Monitoring of Growing Noble Metal Nanostructures Via a Simplified Spatially Confined Growth Method. ACS Appl. Mater. Interfaces 2015, 7, 7516-7525.

(28) Nikoobakht, B.; El-Sayed, M. A. Preparation and Growth Mechanism of Gold Nanorods (NRs) Using Seed-Mediated Growth Method. Chem. Mater. 2003, 15, 1957-1962.

(29) Zheng, Y.; Zhong, X.; Li, Z.; Xia, Y. Successive, Seed-Mediated Growth for the Synthesis of Single-Crystal Gold Nanospheres with Uniform Diameters Controlled in the Range of 5-150 nm. Part. Part. Syst. Charact. 2014, 31, 266-273.
(30) Leopold, N.; Lendl, B. A New Method for Fast Preparation of Highly Surface-Enhanced Raman Scattering (SERS) Active Silver Colloids at Room Temperature by Reduction of Silver Nitrate with Hydroxylamine Hydrochloride. J. Phys. Chem. B 2003, 107, 57235727.

(31) Tan, X.; Wang, Z.; Yang, J.; Song, C.; Zhang, R.; Cui, Y. Polyvinylpyrrolidone- (PVP-) Coated Silver Aggregates for High Performance Surface-enhanced Raman Scattering in Living Cells. Nanotechnology 2009, 20, 445102.

(32) Wu, L.; Wang, Z.; Zong, S.; Huang, Z.; Zhang, P.; Cui, Y. A SERS-Based Immunoassay with Highly Increased Sensitivity Using Gold/Silver Core-Shell Nanorods. Biosens. Bioelectron. 2012, 38, 9499.

(33) Orendorff, C. J.; Gearheart, L.; Jana, N. R.; Murphy, C. J. Aspect Ratio Dependence on Surface Enhanced Raman Scattering Using Silver and Gold Nanorod Substrates. Phys. Chem. Chem. Phys. 2006, 8, 165-170.

(34) Park, H.; Lee, S.; Chen, L.; Lee, E. K.; Shin, S. Y.; Lee, Y. H.; Son, S. W.; Oh, C. H.; Song, J. M.; Kang, S. H.; Choo, J. SERS Imaging of HER2-Overexpressed MCF7 Cells Using Antibody-Conjugated Gold Nanorods. Phys. Chem. Chem. Phys. 2009, 11, 7444-7449.

(35) Nima, Z. A.; Mahmood, M.; Xu, Y.; Mustafa, T.; Watanabe, F.; Nedosekin, D. A.; Juratli, M. A.; Fahmi, T.; Galanzha, E. I.; Nolan, J. P.; Basnakian, A. G.; Zharov, V. P.; Biris, A. S. Circulating Tumor Cell Identification by Functionalized Silver-Gold Nanorods with Multicolor, Super-Enhanced SERS and Photothermal Resonances. Sci. Rep. 2014, 4, 4752.

(36) Xiang, Y. U.; Wu, X. C.; Liu, D. F.; Li, Z. Y.; Chu, W. G.; Feng, L. L.; Zhang, K.; Zhou, W. Y.; Xie, S. S. Gold Nanorod-Seeded Growth of Silver Nanostructures: From Homogeneous Coating to Anisotropic Coating. Langmuir 2008, 24, 3465-3470.

(37) Abalde-Cela, S.; Aldeanueva-Potel, P.; Mateo-Mateo, C.; Rodriguez-Lorenzo, L.; Alvarez-Puebla, R. A.; Liz-Marzan, L. M. Surface-Enhanced Raman Scattering Biomedical Applications of Plasmonic Colloidal Particles. J. R. Soc., Interface 2010, 7, S435-S450.

(38) Wang, H.; Zhao, X.; Meng, W.; Wang, P.; Wu, F.; Tang, Z.; Han, X.; Giesy, J. P. Cetyltrimethylammonium Bromide-Coated $\mathrm{Fe}_{3} \mathrm{O}_{4}$ Magnetic Nanoparticles for Analysis of 15 Trace Polycyclic Aromatic Hydrocarbons in Aquatic Environments by Ultraperformance, Liquid Chromatography with Fluorescence Detection. Anal. Chem. 2015, 87, $7667-7675$.

(39) Khanal, B. P.; Zubarev, E. R. Rings of Nanorods. Angew. Chem., Int. Ed. 2007, 46, 2195-2198.

(40) Kneipp, J.; Kneipp, H.; Wittig, B.; Kneipp, K. Following the Dynamics of $\mathrm{Ph}$ in Endosomes of Live Cells with SERS Nanosensors. J. Phys. Chem. C 2010, 114, 7421-7426.

(41) Talley, C. E.; Jusinski, L.; Hollars, C. W.; Lane, S. M.; Huser, T. Intracellular Ph Sensors Based on Surface-Enhanced Raman Scattering. Anal. Chem. 2004, 76, 7064-7068.

(42) Krug, H. F. Nanosafety Research-Are We on the Right Track? Angew. Chem., Int. Ed. 2014, 53, 12304-12319.

(43) Ostermeyer, A. K.; Kostigen Mumuper, C.; Semprini, L.; Radniecki, T. Influence of Bovine Serum Albumin and Alginate on Silver Nanoparticle Dissolution and Toxicity to Nitrosomonas Europaea. Environ. Sci. Technol. 2013, 47, 14403-14410.

(44) Loza, K.; Diendorf, J.; Sengstock, C.; Ruiz-Gonzalez, L.; Gonzalez-Calbet, J. M.; Vallet-Regi, M.; Köller, M.; Epple, M. The Dissolution and Biological Effects of Silver Nanoparticles in Biological Media. J. Mater. Chem. B 2014, 2, 1634-1643.

(45) Li, L.; Wu, H.; Peijnenburg, W. J.; van Gestel, C. A. Both Released Silver Ions and Particulate Ag Contribute to the Toxicity of AgNPs to Earthworm Eisenia Fetida. Nanotoxicology 2015, 9, 792801.

(46) Wang, X.; Ji, Z.; Chang, C. H.; Zhang, H.; Wang, M.; Liao, Y. P.; Lin, S.; Meng, H.; Li, R.; Sun, B.; Winkle, L. V.; Pinkerton, K. E.; Zink, J. I.; Xia, T.; Nel, A. E. Use of Coated Silver Nanoparticles to Understand the Relationship of Particle Dissolution and Bioavailability to Cell and Lung Toxicological Potential. Small 2014, 10, 385-398. 
(47) Wang, Y.; Chen, L.; Liu, P. Biocompatible Triplex Ag@SiO $\mathrm{mTiO}_{2}$ Core-Shell Nanoparticles for Simultaneous Fluorescence-SERS Bimodal Imaging and Drug Delivery. Chem.-Eur. J. 2012, 18, 59355943. 